\title{
Impact of Strong Raman Self-Frequency Shift on Bound State of Dissipative Solitons
}

\author{
Cong Xu \\ Department of Physics, Sichuan Normal University, No. 5 Jing'an Road, Chengdu 610068, China \\ Correspondence should be addressed to Cong Xu; xucong017@qq.com
}

Received 11 September 2015; Accepted 3 November 2015

Academic Editor: Vasily Spirin

Copyright (C) 2015 Cong Xu. This is an open access article distributed under the Creative Commons Attribution License, which permits unrestricted use, distribution, and reproduction in any medium, provided the original work is properly cited.

\begin{abstract}
Bound dissipative solitons are numerically studied by implementing strong Raman self-frequency shift (RSFS) in an all-normaldispersion (ANDi) Yb-doped fiber laser. Results demonstrated that overstrong RSFS had no filter-like effect in the ANDi fiber laser when a bandpass filter was present in the intracavity. However, overstrong RSFS could cause the bandpass filter to destabilize the ANDi fiber laser. For the first time in the field, we have demonstrated that strong RSFS could destabilize bound DS pulses and generate noise-like bound pulses. Furthermore, the generation mechanism of destabilized noise-like bound pulses in the fiber laser with intracavity filter is different from the noise-like pulses in the fiber lasers without a bandpass filter.
\end{abstract}

\section{Introduction}

Increasing pulse energy will result in pulse breaking because excessive nonlinear phase shift will occur due to anomalous dispersion in the fiber laser. The generation of energy pulses has resulted in the development of various methods that aimed to overcome limitations of traditional soliton pulses imposed by overlarge nonlinear intracavity. Dissipative soliton (DS) is one of the most effective ways to achieve high energy pulses [1]. DSs are generated in normal dispersion mode-locking fiber laser and have been of interest to researchers for years because of the application of wide spectrum bandwidths and high pulse energies [1].

Different fiber laser operation regimes can be achieved in suitable parameter conditions, namely, soliton-bunching, stable soliton distribution, and stochastic soliton motion modes [2]. In some cases, multipulses can be tightly bound together with discrete, fixed-pulse separations [3], such as single DS [1], multicolor bound DS [4], and multibound DS [5]. Practical and theoretical studies of bound DS have attracted great attention [6-8]. Multipulse or multibound DS formation is usually regarded as a result of strong pumping, and the pulse peak clamping effect is induced in the cavity $[2,9]$. However, excessive nonlinearity induced by strong pumping tends to destroy output pulses regardless of fiber laser operation mode [10, 11]. When gain level increases, the stability of single DS mode would be destroyed by ASE-noise [12]. Excessive nonlinearity and dispersion in a cavity destabilize bound solitons and lead to soliton bunch formation [13].

Another study has demonstrated that unstable bound states can be achieved when finite relaxation time of the saturable absorber is considered in the simulation [5]. However, the stability of bound DS about RSFS or other high order nonlinearity effects in fiber laser have not been rigorously studied, but the Raman effect that modifies the group velocity of a bound state of solitons differently from that of a single soliton has been reported [14]. Raman pulse (RP) or even Raman dissipative solitons (RDS) can be generated in the presence of strong stimulated Raman scattering (SRS) [15]. The noisy Raman pulse is another fundamental limit of the energy and stability of DS [16]. However, these studies are based on single DS or multicolor bound DS regime [17], but not on bound DS regime.

This study reports the stability of bound DSs in ANDi fiber laser in the presence of both strong RSFS and theoretical filtering. Stable bound DS can be generated in the ANDi fiber laser with a certain filter, but strong RSFS can make noiselike bound pulses instead of stable bound DS. Furthermore, when ANDi fiber laser is working in single pulse state, strong 


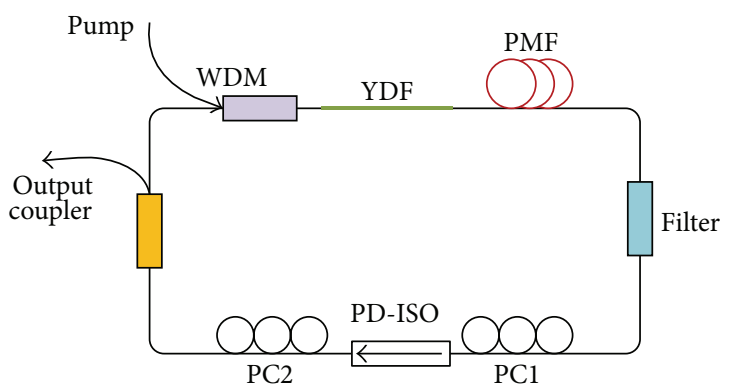

FIGURE 1: Setup of simulation model. WDM: wavelength-division multiplexer; PC: polarization controller; PD-ISO: polarizationdependent isolator; YDF: Yb-doped fiber; and PMF: polarizationmaintaining optical fiber.

RSFS can also make the output DS unstable. This kind of unstable pulse is a noise-like bound pulse and is different from that in ANDi-fiber laser without filter. The mechanism of the generation of bound noise-like pulse is the Raman induced noise caused by strong RSFS, and not by ASE-noise and strong SPM within the resonant.

\section{Numerical Simulation Model}

Research on bound DS is based on gain guided soliton fiber lasers, but not all-normal-dispersion fiber laser $[2,18]$. In these studies, the gain curves of EDF have a bandwidth of about $16 \mathrm{~nm}$, which is narrower than YDF (about 40 to $45 \mathrm{~nm}$ ). Hence, a filter should be used in the ANDi fiber laser within the resonant to generate bound DS. To perform this step, we used a simulation model, as shown in Figure 1.

Our numerical simulation is performed using a generalized nonlinear Schrödinger equation (NLSE) [19], which has RSFS. Given its long cavity length, ANDi fiber laser can generate DS pulse output, and Raman pulse can be generated when long PMF is used intracavity [4]. Consider

$$
\begin{array}{r}
\frac{\partial A}{\partial z}+\frac{\alpha}{2} A+\frac{i \beta_{2}}{2} \frac{\partial^{2} A}{\partial T^{2}}-\frac{\beta_{3}}{6} \frac{\partial^{3} A}{\partial T^{3}} \\
\quad=i \gamma\left[|A|^{2} A-T_{R} A \frac{\partial|A|^{2}}{\partial T}\right] .
\end{array}
$$

Equation (1) is valid for simulating pulse propagation in passive fiber with RSFS effect. The electric field envelope of the intracavity pulse is represented by $A(z, t)$. The secondand third-order dispersion coefficients are $\beta_{2}$ and $\beta_{3}$ of PMF, which are used as $\beta_{2}=47 \mathrm{ps}^{2} / \mathrm{km}$ and $\beta_{3}=0.013 \mathrm{ps}^{3} / \mathrm{km}$, respectively. The nonlinearity coefficient of the fiber is denoted by $\gamma$, whereas the Raman time constant $T_{R}$ determines the effects of RSFS. $T_{R}$ is the first moment of nonlinear response function [19]. We assumed that $T_{R}$ changes linearly when frequency is near $\omega_{0}=1060 \mathrm{~nm}$. The typical value of $T_{R}$ is $5 \mathrm{fs}$.
Equation (1) describes the pulse propagation in the PMF. Equation (2) (NLSE with gain and without RSFS) describes the pulse propagation in the gain fiber:

$$
\frac{\partial A}{\partial z}+\frac{\alpha}{2} A+\frac{i \beta_{2}}{2} \frac{\partial^{2} A}{\partial T^{2}}-\frac{\beta_{3}}{6} \frac{\partial^{3} A}{\partial T^{3}}=i \gamma|A|^{2} A+\frac{g}{2} A .
$$

The gain fiber has parabolic frequency dependence of $40 \mathrm{~nm}$ bandwidth. The second- and third-order dispersion coefficients are $23 \mathrm{ps}^{2} / \mathrm{km}$ and $-0.013 \mathrm{ps}^{3} / \mathrm{km}$, respectively. The gain saturation of YDF is modeled as follows:

$$
g(z, t)=g_{0} \frac{1}{1+E / E_{\mathrm{sat}}},
$$

where $g_{0}$ is small signal gain, $E_{\text {sat }}$ is gain saturation energy, and $E$ is pulse energy. We use Gaussian bandpass filter in the simulation model with a bandwidth of $16.3 \mathrm{~nm}$. Two PCs and a PD-ISO are used to obtain NPE-based mode-locking within the resonant and are described as follows:

$$
q(z, t)=\frac{q_{0}}{1+|A(z, t)|^{2} / P_{\text {Sat }}},
$$

where $q(z, t)$ characterizes the absorption of NPE-based fast saturable absorber, $P_{\text {Sat }}=50 \mathrm{~W}$ is saturation power, and $q_{0}=$ 0.7 is unsaturated loss.

All other parameters used in the simulations are as follows. The length of YDF is $2 \mathrm{~m}$ and the PMF is $40 \mathrm{~m}$. The length of SMF is ignored. Loss caused by output coupler is $10 \%$. The nonlinearity coefficients in YDF and PMF are $5 \mathrm{~m}^{-1} \mathrm{~W}^{-1}$. The $E_{\text {sat }}$ of YDF is $1000 \mathrm{pJ}$.

\section{Results and Discussions}

Based on the simulation model in Section 2, we obtained numerical results without RSFS intracavity. Figure 2 shows the generated output pulses when the small signal gain is set to $1 \mathrm{~m}^{-1}$ and $3 \mathrm{~m}^{-1}$.

Single pulse mode-lock state can be generated when $g_{0}=$ $1 \mathrm{~m}^{-1}$. Filter intracavity has two effects, namely, filtering out ASE-noise to make the DS pulse stable and attenuating the side parts of the DS pulse spectrum to act as SA. The peak power and energy of the output pulse, as well as the spectrum bandwidth, would increase when $g_{0}$ is increasing.

However, when $g_{0}=3 \mathrm{~m}^{-1}$, typical bound solitons can be generated, as shown in Figures 2(c) and 2(d). The two DS pulses in Figure 2(c) have almost the same pulse energies and temporal widths. Moreover, both pulses have a constant pulse spacing of $210 \mathrm{ps}$, which is almost random in each simulation. Pulse splitting can keep the peak power intracavity at a lower status. The spectrum of output pulse is very different from single DS pulse state, as shown in Figures 2(b) and 2(d).

The optical spectrum in Figure 2(d) has clear spectral modulations, which are similar to the study of Zhao et al. $[2,3]$. Unlike in multicolor and harmonic mode-locking DSs, pulse spacing is kept constant (about 210 ps) during simulation. Thus, we ensured that the pulses we simulated are in the bound state. In addition, the spectrum base in Figure 2(d) is different from the single DS state in Figure 2(b), 


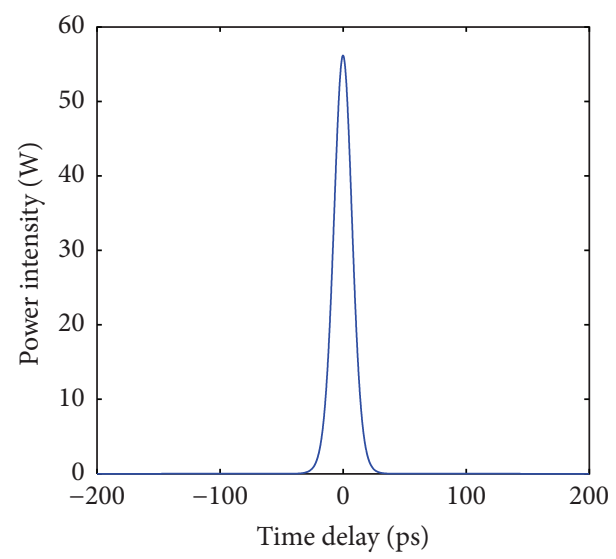

(a)

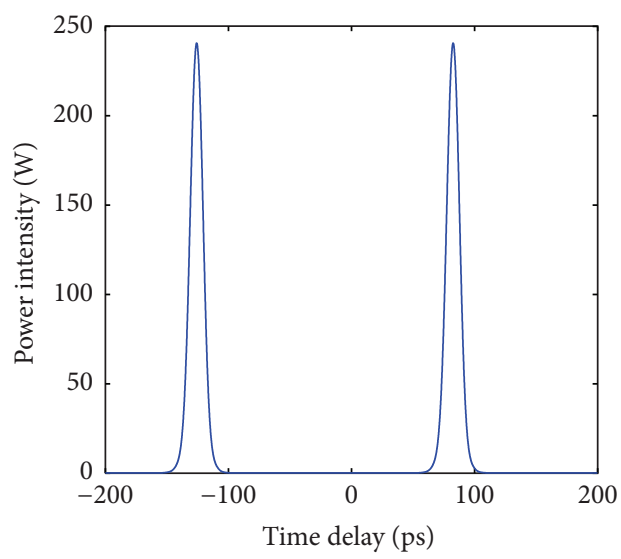

(c)

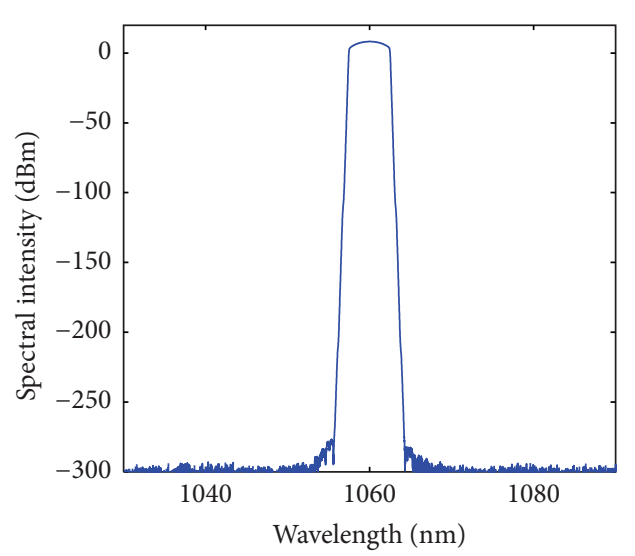

(b)

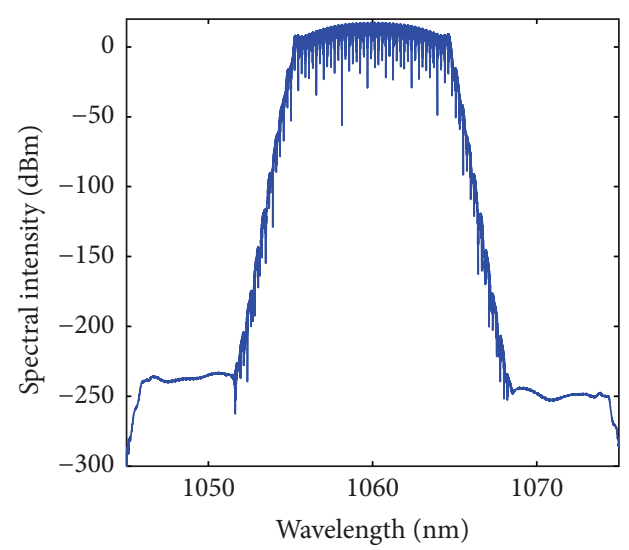

(d)

FIGURE 2: Temporal and spectral envelopes of single DS pulse with gain of $1 \mathrm{~m}^{-1}((\mathrm{a}),(\mathrm{b}))$ and bound DS pulses with gain of $3 \mathrm{~m}^{-1}((\mathrm{c}),(\mathrm{d}))$.

and the spectral base in long-wavelength side is lower by about $10 \mathrm{~dB}$ than the short-wavelength side.

The number of intracavity pulses increases as the gain increases. The peak power intracavity will be lower than the single pulse state because of this increase. Hence, nonlinearity is restricted at a lower range. Furthermore, spectrum width would be broadened because of increased pump power.

With all other parameters held constant, we simulated pulse operation intracavity with RSFS. Figure 3 shows the results.

The spectrum of output pulses changes with RSFS enhancement when the gain is $2 \mathrm{~m}^{-1}$. As shown in Figures $3(\mathrm{f})$ and $3(\mathrm{~g})$, the spectrum base of the long-wavelength side is pumped by the short-wavelength side, which is a typical characteristic of RSFS [20]. After 10,000 loops, the shortwavelength side is still narrower and lower than the longwavelength side, and the output pulse has a stable pulse shape. Previous research indicated that RSFS can provide the passive mode-lock ANDi fiber laser with less self-start time and the RSFS acts as a high pass filter at the rising edge of the pulse [21]. However, unlike the wavelength without filter in [20], central wavelength does not change because of the bandpass filter.

When the RSFS ( $T_{R}=55 \mathrm{fs}$ ) in Figures 3(c) and 3(g) was enhanced, unstable pulse base was found in output pulses.
The unstable part continued to exist even after 30,000 loops (Figure 3(c)). Considering the existence of filter intracavity, the spikes at the long-wavelength side were caused by strong RSFS, and the unstable pulse front can be attributed to normal dispersion intracavity and the linear chirp, which composes the long-wavelength components of DS in the front of the pulse.

When the Raman time constant is set as $T_{R}=100 \mathrm{fs}$, the output pulse became unstable. This finding is similar to the noise-like pulse. The amount of output pulse is 3, which indicated that the fiber laser was operating in multipulse state. Pulse spacing was kept constant (about $100 \mathrm{ps}$ ) during our simulation, and the spectrum exhibited spectral modulations. This finding implied that output pulse could be identified as a bound soliton. However, unlike in the bound DS pulses in Figure 2(c), the pulses in Figure 3(d) are unstable during simulation. The noiselike bound pulse is also different from traditional noise-like pulse in [12] because of the amount of pulse. Even when the gain is larger (e.g., $2.5 \mathrm{~m}^{-1}$ ), stable bound DS pulses can be changed into unstable noise-like bound pulse, as shown in Figure 4. Figure 4 shows that strong RSFS can make the bound DS pulses unstable, and the generated unstable noise-like bound pulse is almost similar to that in Figure 3. 


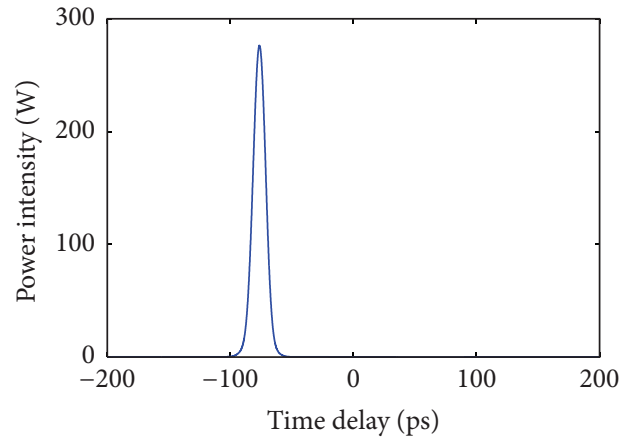

(a)

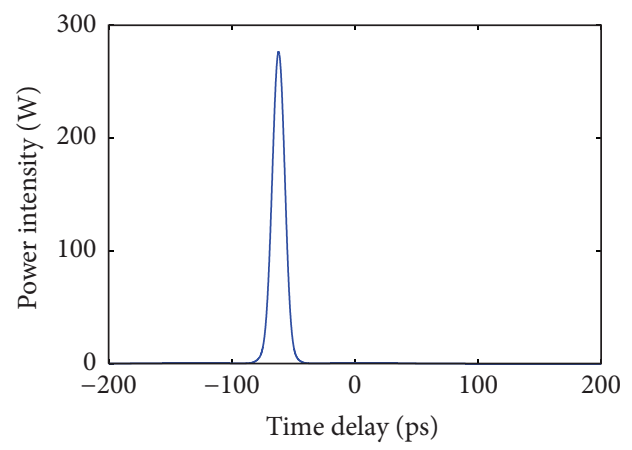

(b)

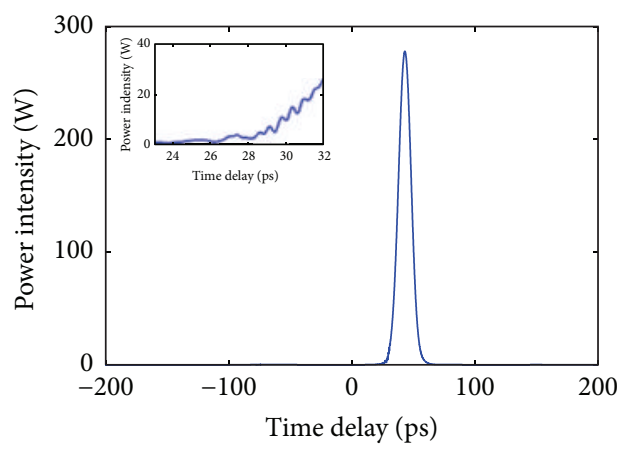

(c)

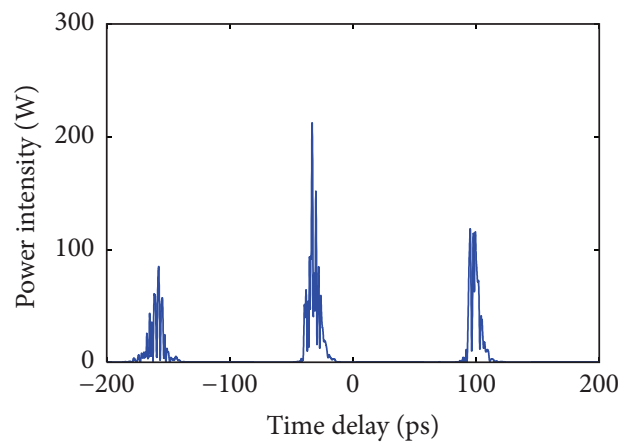

(d)

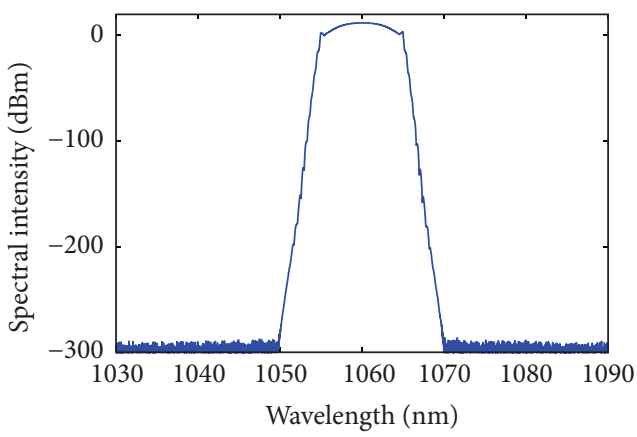

(e)

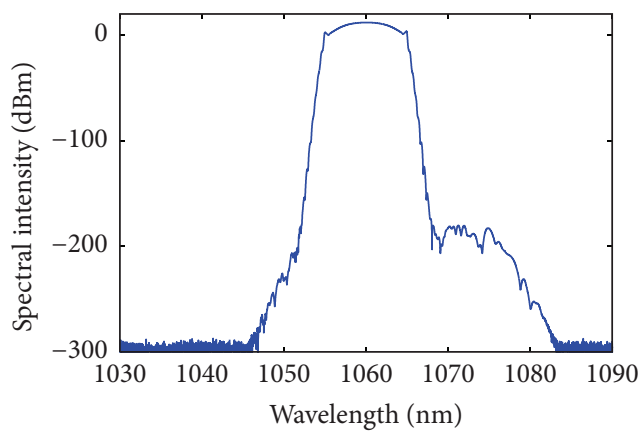

(f)

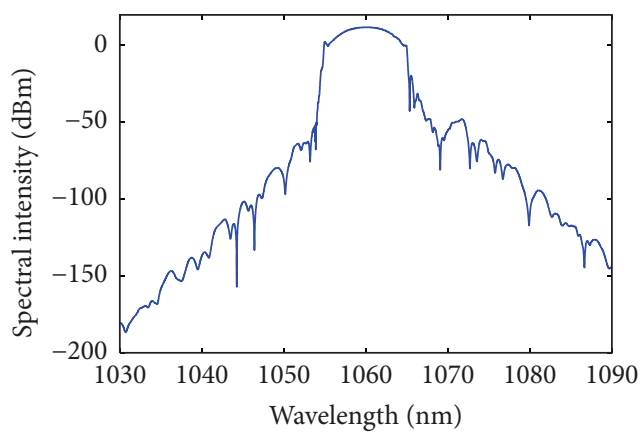

(g)

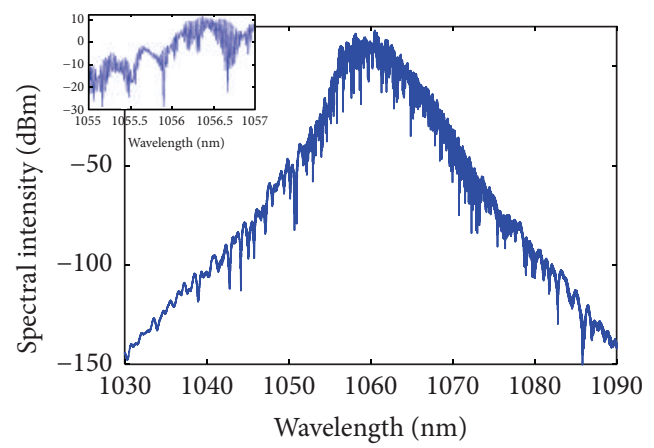

(h)

Figure 3: Temporal and spectral envelopes of stable DS and noise-like bound pulses, with different Raman time constants when gain is $2 \mathrm{~m}^{-1}$. ((a), (e)) No RSFS; ((b), (f)) $T_{R}=50 \mathrm{fs;} \mathrm{((c),} \mathrm{(g))} T_{R}=85 \mathrm{fs}$; ((d), (h)) $T_{R}=100 \mathrm{fs}$. Insert: (c) front side part temporal profile of $T_{R}=85 \mathrm{fs}$; (h) details of the spectrum of $T_{R}=100 \mathrm{fs}$. 


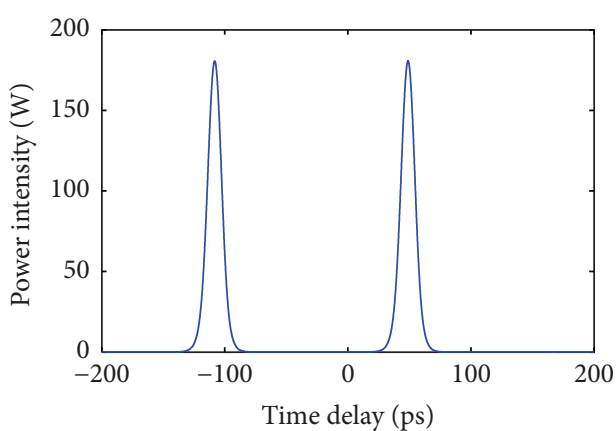

(a)

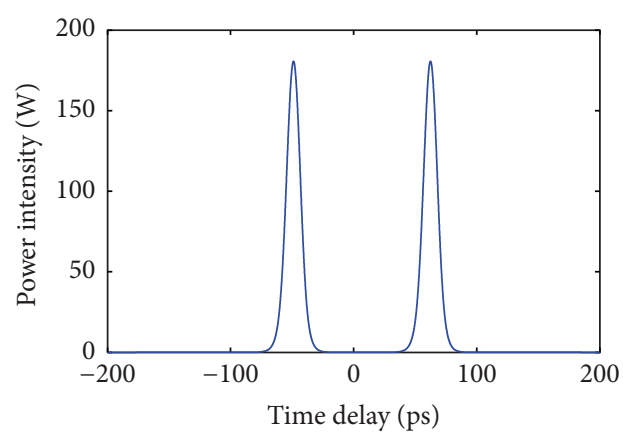

(b)

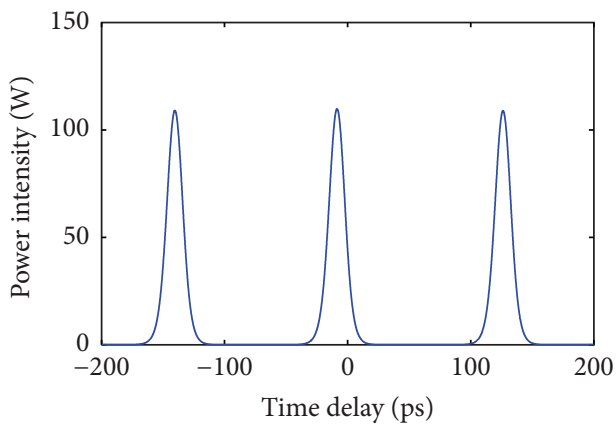

(c)

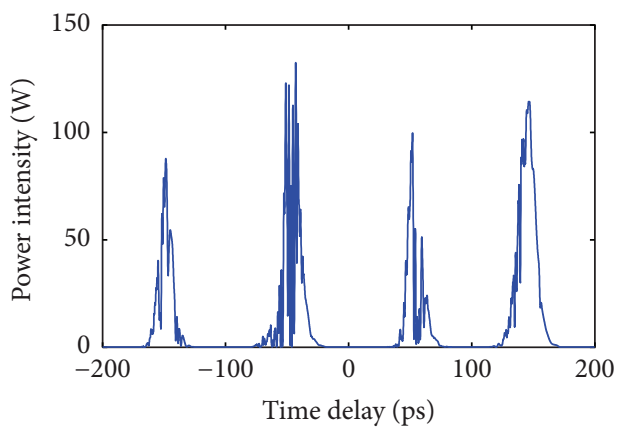

(d)

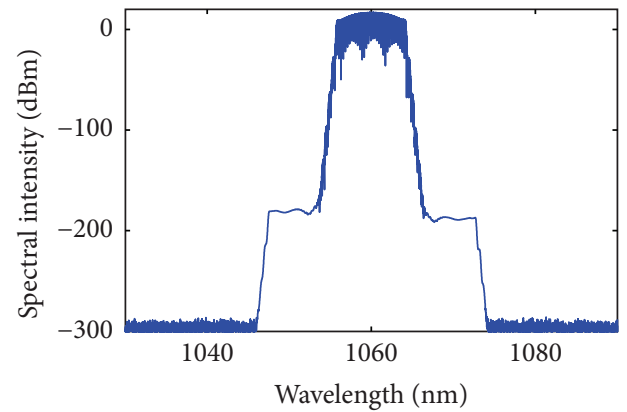

(e)

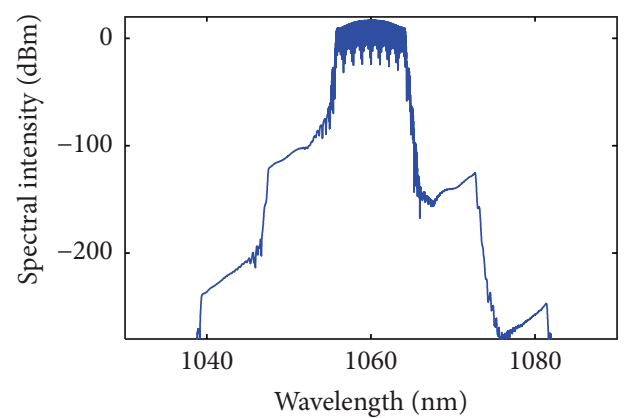

(f)

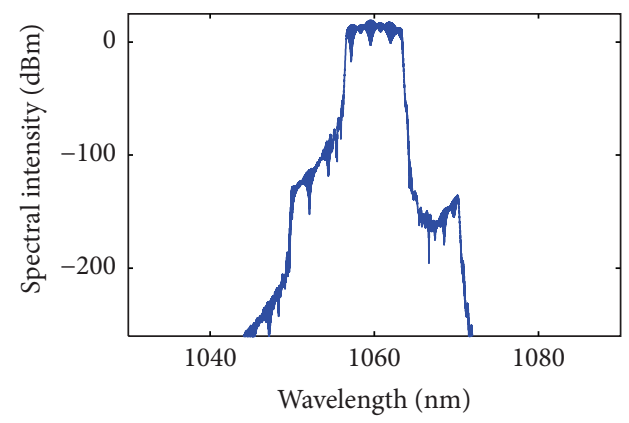

(g)

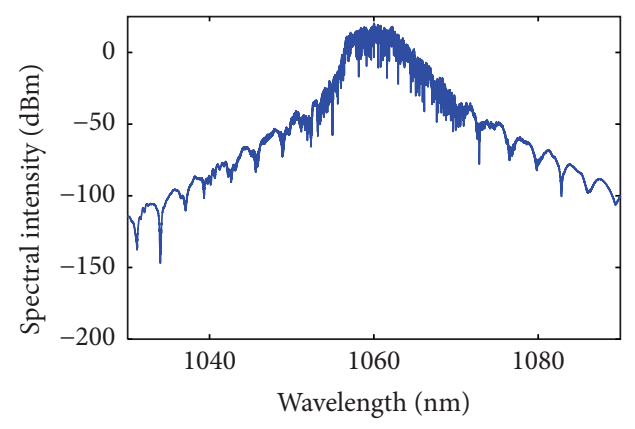

(h)

FIGURE 4: Temporal and spectral envelopes of stable DS and noise-like bound pulses, with different Raman time constants when gain is $2.5 \mathrm{~m}^{-1}$. ((a), (e)) No RSFS; ((b), (f)) $T_{R}=25 \mathrm{fs}$; ((c), (g)) $T_{R}=50 \mathrm{fs} ;((\mathrm{d}),(\mathrm{h})) T_{R}=75 \mathrm{fs}$.

Moreover, the spectral sideband in Figures 4(f) and 4(g) is affected by the enhanced RSFS. Compared with those in Figures 2(d) and 4(e), the long-wavelength side is raised and the short-wavelength side is suppressed.
In [21], traditional noise-like pulse is caused by overlarge ASE-noise in gain fiber. However, in our simulation, the band pass filter intracavity can suppress ASE-noise and stabilize the output DS pulse [12]. Hence, the cause of unstable pulses 


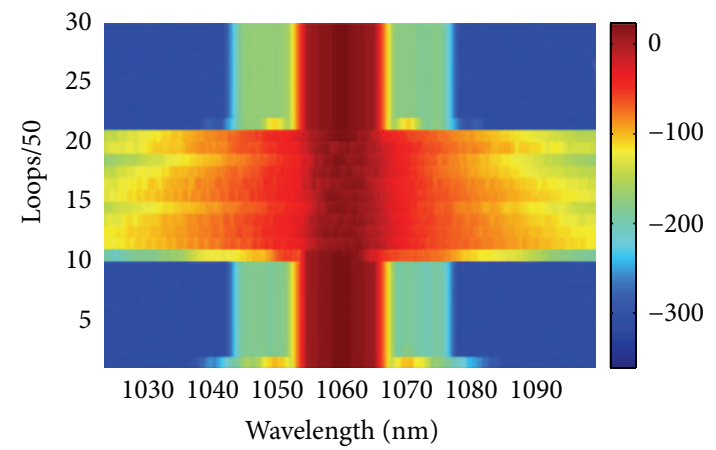

(a)

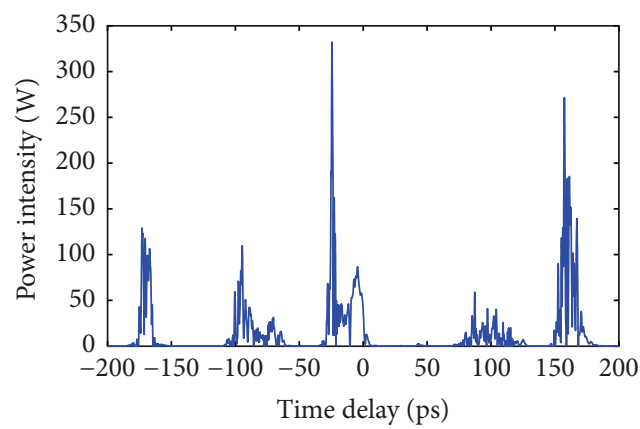

(c)

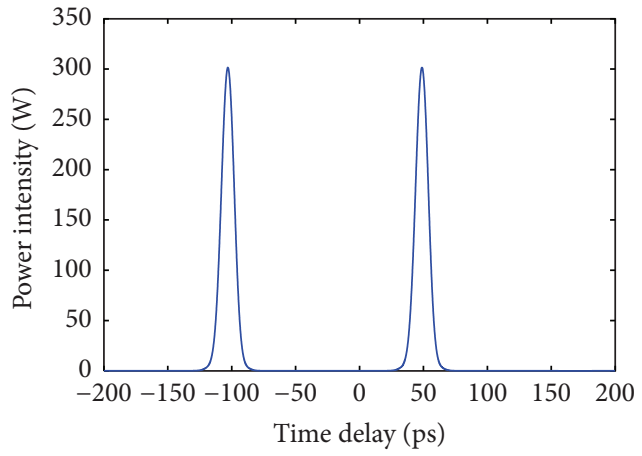

(b)

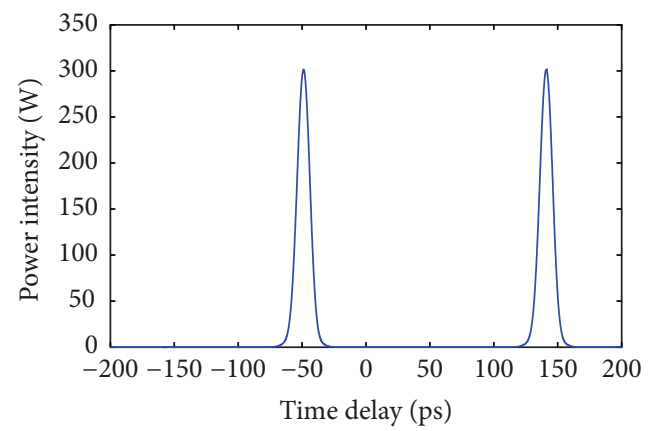

(d)

FIGURE 5: Pulse evolution process of ANDi fiber laser, with different Raman time constants during simulation. (a) Spectrum evolution process; (b) temporal profile of 400th loop; (c) temporal profile of 900th loop; (d) temporal profile of 1400th loop.

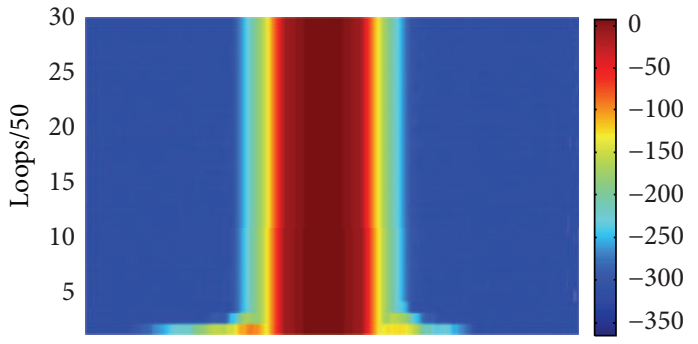

1030104010501060107010801090 Wavelength (nm)

(a)

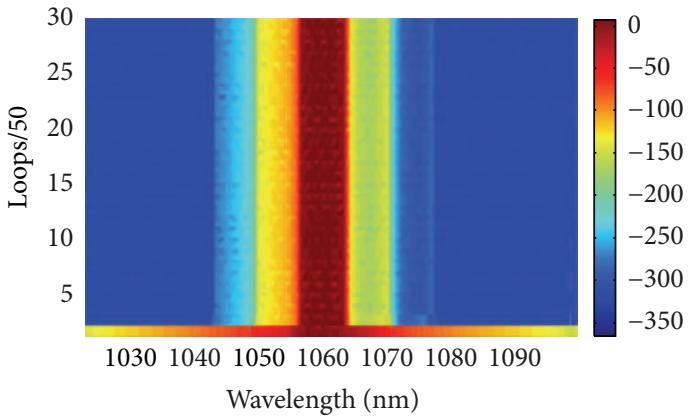

(c)

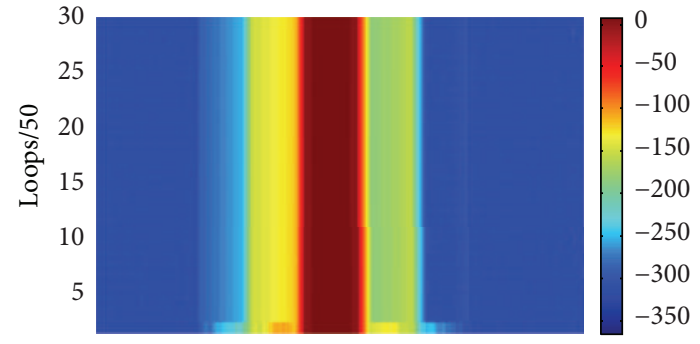

1030104010501060107010801090 Wavelength $(\mathrm{nm})$

(b)

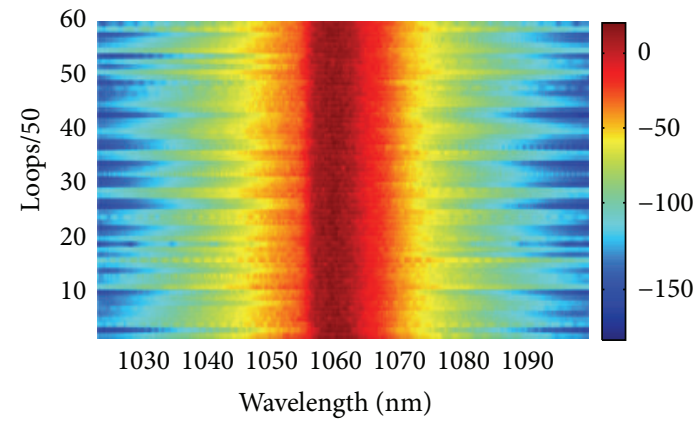

(d)

FIgURE 6: Pulse spectrum evolution processes of ANDi fiber laser at different Raman time constants. (a) No RSFS; (b) $T_{R}=25 \mathrm{fs}$; (c) $T_{R}=50 \mathrm{fs}$; (d) $T_{R}=75 \mathrm{fs}$. 


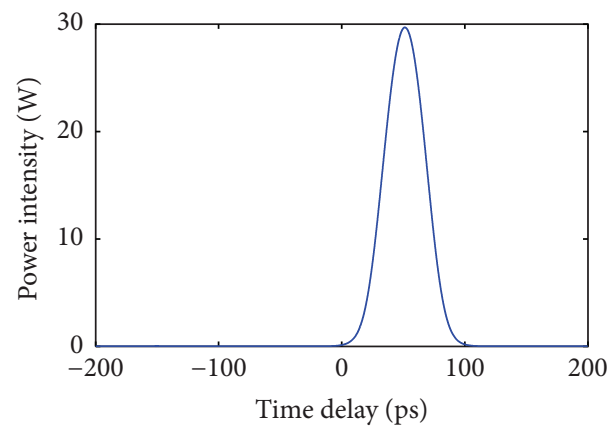

(a)

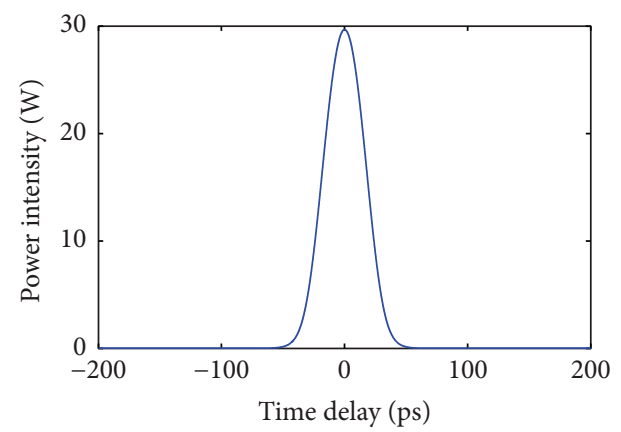

(b)

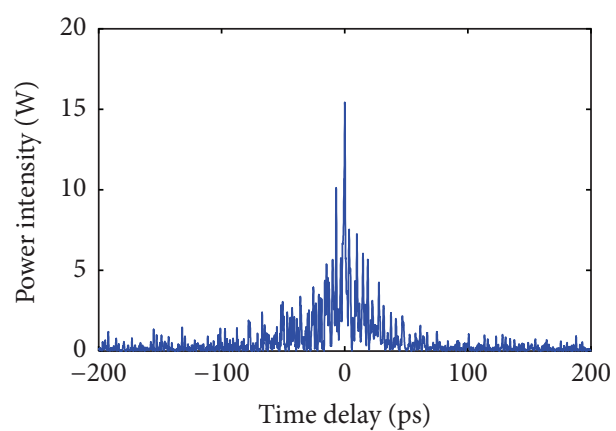

(c)

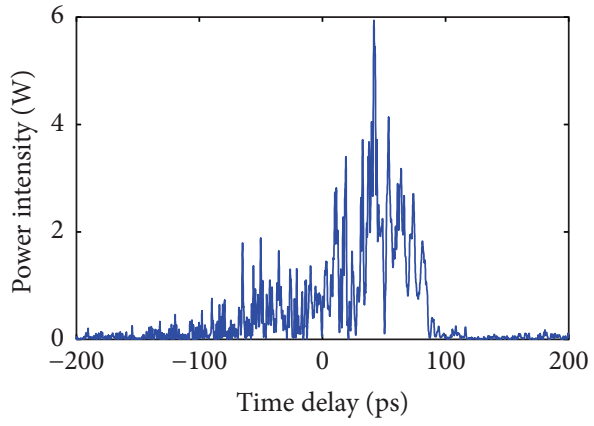

(d)

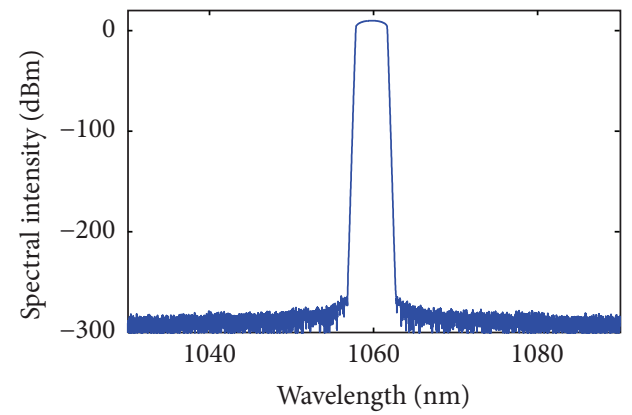

(e)
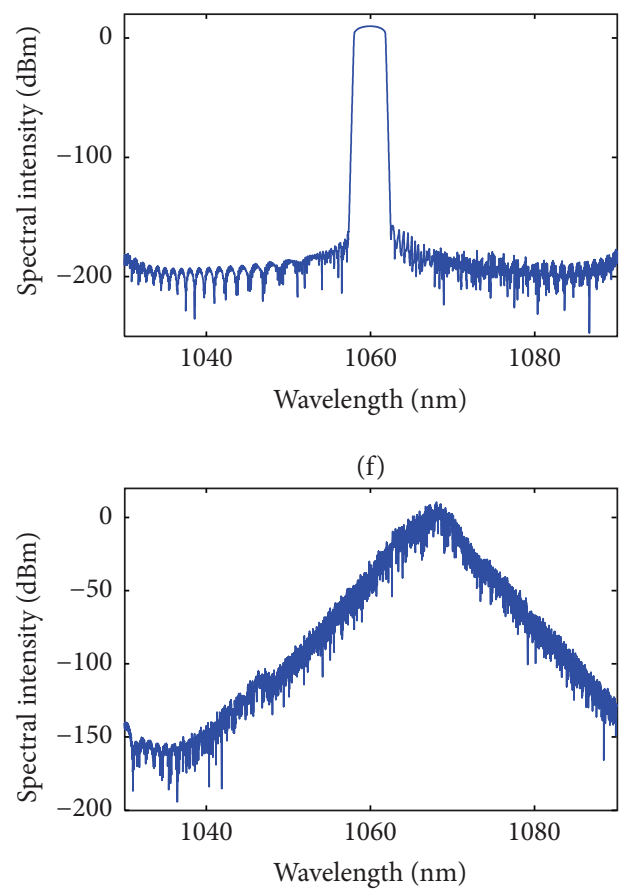

(g)

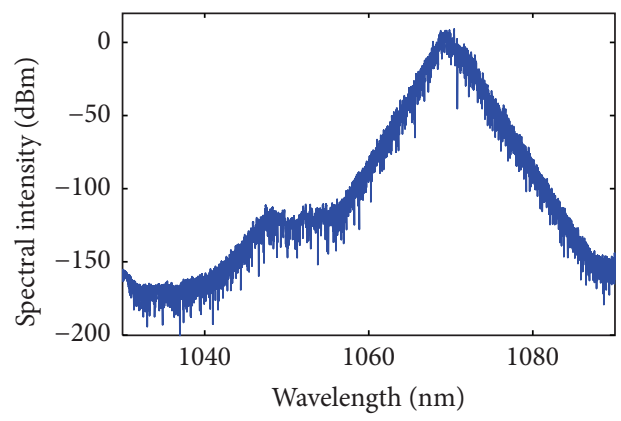

(h)

FIGURE 7: Temporal and spectral envelopes of stable DS and noise-like bound pulses without filter intracavity with different Raman time constants when gain is $2.5 \mathrm{~m}^{-1}$. ((a), (e)) No RSFS; ((b), (f)) $T_{R}=25 \mathrm{fs} ;((\mathrm{c}),(\mathrm{g})) T_{R}=50 \mathrm{fs}$; ((d), (h)) $T_{R}=100 \mathrm{fs}$.

would be regarded as other effects. Figure 3 shows that the output pulse is stable when RSFS is not strong. Thus, we can assume that strong RSFS causes noise-like bound pulses.

To verify this assumption, we perform the following simulation. RSFS is not considered during the first 500 loops.
However, the RSFS of $T_{R}=75 \mathrm{fs}$ would be taken into account in the next 500 loops and followed by another 500 loops for $T_{R}=0$. Figure 5 shows the simulation results. When RSFS does not participate in the simulation, the output pulse would be stable after the first 30 loops and the bound soliton 
mode-lock state is achieved. However, unstable noise-like bound pulses were generated in the 500 to 1000 loops. The stable pulse will not be achieved until $T_{R}$ is reset to 0 during the 1000 to 1500 loops.

Depending on these simulations, strong RSFS is the key factor of the noise-like bound DS pulse generation. Figure 3 shows that strong RSFS could pump energy from the shortwavelength side to the long-wavelength side. Spectrum spikes would then be generated in the long-wavelength side. Given the large normal dispersion of fiber laser, the newly generated frequency components could be found at the front side of the pulse, which would make the pulse envelop unsmooth. Considering the loss caused by the bandpass filter, the unsmooth front side part in Figure 3(c) could be regarded as a Raman-driven destabilization similar to that in [16]. The amplified RP-like part would break the nonlinearity filtering balance and change bound solitons into noise-like bound pulses.

Furthermore, we used different $T_{R}$ in the simulation to compare the starting process of the laser. The results are shown in Figure 6.

Figure 6 shows that more iteration loops are needed to make the pulse stable when the Raman time constants are increasing. When $T_{R}$ is larger than a certain threshold value, such as $90 \mathrm{fs}$ in this simulation, stable pulses would not be obtained even after $10^{5}$ loops. However, when no filter intracavity is present, the central wavelength and the starting time of the laser would all change [21]. Thus, we enhanced the Raman time constants, as shown in Figures $7(a), 7(b)$, $7(\mathrm{c})$, and $7(\mathrm{~d})$. The amplitude of the output pulse is lower than that without RSFS. The central wavelength in Figures $7(\mathrm{~g})$ and 7(h) has obviously shifted. The parabolic gain curve of YDF could provide the pulse with a lower gain, which is different from the noise-like bound pulse. Filter intracavity reduced the central wavelength shift and suppressed ASE-noise.

Based on the calculations, strong RSFS can make the back side of the pulse smooth when no filter is used. However, when a filter is used in the fiber laser, the filter-like effect would be replaced by the bandpass filter. The Raman frequency components in the front side would make the pulse unstable. Hence, when a filter is present in the ANDi-fiber laser, strong RSFS can counteract some of the effects of the bandpass filter and make the bound DS pulses unstable.

\section{Conclusion}

We demonstrated that strong RSFS could make bound DS pulses unstable even when a bandpass filter is used in an ANDi fiber laser for the first time. Theoretically, unstable pulses are characterized by noise-like and bound DS pulse. Strong RSFS can make the ANDi-fiber laser have a long self-starting time. Depending on simulation, strong RSFS in ANDi fiber laser can cause pulses to have higher resistance against ASE-noise only when the bandpass filter is absent. In conclusion, overstrong RSFS should be avoided only when bound soliton is going to be generated. Suitable RSFS can make the single DS output ANDi fiber laser stable with shorter self-starting time when no filter is used in the fiber laser.

\section{Conflict of Interests}

The author declares that there is no conflict of interests regarding the publication of this paper.

\section{Acknowledgments}

This work was supported in part by National 973 Program (2012CB315705), NSFC Program (61475105, 61271042, 61107058,61205079 , and 61302016), China Postdoctoral Science Foundation (2013M540891), NCET-13-0682, and the Science Research Fund of the Sichuan Provincial Education Department under Grant 14ZB0041.

\section{References}

[1] S. Kobtsev, S. Kukarin, S. Smirnov, S. Turitsyn, and A. Latkin, "Generation of double-scale femto/pico-second optical lumps in mode-locked fiber lasers," Optics Express, vol. 17, no. 23, pp. 20707-20713, 2009.

[2] D. Y. Tang, L. M. Zhao, B. Zhao, and A. Q. Liu, "Mechanism of multisoliton formation and soliton energy quantization in passively mode-locked fiber lasers," Physical Review A, vol. 72, no. 4, Article ID 043816, 2005.

[3] L. M. Zhao, D. Y. Tang, T. H. Cheng, H. Y. Tam, and C. Lu, "Bound states of dispersion-managed solitons in a fiber laser at near zero dispersion," Applied Optics, vol. 46, no. 21, pp. 47684773, 2007.

[4] S. A. Babin, E. V. Podivilov, D. S. Kharenko et al., "Multicolour nonlinearly bound chirped dissipative solitons," Nature Communications, vol. 5, article 4653, 2014.

[5] A. Zaviyalov, R. Iliew, O. Egorov, and F. Lederer, "Multi-soliton complexes in mode-locked fiber lasers," Applied Physics B: Lasers and Optics, vol. 104, no. 3, pp. 513-521, 2011.

[6] D. Y. Tang, W. S. Man, H. Y. Tam, and P. D. Drummond, "Observation of bound states of solitons in a passively modelocked fiber laser," Physical Review A, vol. 64, no. 3, Article ID 033814, 2001

[7] L. Yun and D. Han, "Bound state of dissipative solitons in a nanotube-mode-locked fiber laser," Optics Communications, vol. 313, pp. 70-73, 2014.

[8] A. K. Komarov, K. P. Komarov, and D. V. Meshcheryakov, "Bound states of dissipative solitons in fiber lasers with a lumped saturable absorber," Optoelectronics, Instrumentation and Data Processing, vol. 47, no. 6, pp. 602-607, 2011.

[9] L. M. Zhao, D. Y. Tang, T. H. Cheng, H. Y. Tam, and C. Lu, "Generation of multiple gain-guided solitons in a fiber laser," Optics Letters, vol. 32, no. 11, pp. 1581-1583, 2007.

[10] S. Smirnov, S. Kobtsev, S. Kukarin, and A. Ivanenko, "Three key regimes of single pulse generation per round trip of all-normaldispersion fiber lasers mode-locked with nonlinear polarization rotation," Optics Express, vol. 20, no. 24, pp. 27447-27453, 2012.

[11] S. Kobtsev, S. Smirnov, S. Kukarin, and S. Turitsyn, "Modelocked fiber lasers with significant variability of generation regimes," Optical Fiber Technology, vol. 20, no. 6, pp. 615-620, 2014. 
[12] C. Xu, Y. T. Dai, Z. X. Zhang et al., "Unstable nonlinear spectral filtering balance in ANDi passive modelocking fiber lasers," in Proceedings of the 23rd Annual Meeting of the IEEE Photonics Society, pp. 673-674, IEEE, Denver, Colo, USA, November 2010.

[13] R. Gumenyuk and O. G. Okhotnikov, "Multiple solitons grouping in fiber lasers by dispersion management and nonlinearity control," Journal of the Optical Society of America B: Optical Physics, vol. 30, no. 4, pp. 776-781, 2013.

[14] M. Olivier, V. Roy, and M. Piché, "Influence of the Raman effect on bound states of dissipative solitons," Optics Express, vol. 14, no. 21, pp. 9728-9742, 2006.

[15] A. E. Bednyakova, S. A. Babin, D. S. Kharenko et al., "Evolution of dissipative solitons in a fiber laser oscillator in the presence of strong Raman scattering," Optics Express, vol. 21, no. 18, pp. 20556-20564, 2013.

[16] C. Aguergaray, A. Runge, M. Erkintalo, and N. G. R. Broderick, "Raman-driven destabilization of mode-locked long cavity fiber lasers: fundamental limitations to energy scalability," Optics Letters, vol. 38, no. 15, pp. 2644-2646, 2013.

[17] S. Kobtsev, S. Kukarin, S. Smirnov, and Y. Fedotov, "Ultra-widetunable fibre source of femto-and picosecond pulses based on intracavity Raman conversion," in Fiber Lasers VII: Technology, Systems, and Applications, vol. 7580 of Proceedings of SPIE, International Society for Optics and Photonics, San Francisco, Calif, USA, January 2010.

[18] Z. Xin, H. Minglie, S. Youjian, C. Lu, and W. Qingyue, "Bound state operations in stretched-pulse mode-locking large-modearea photonic-crystal-fiber laser," Acta Optica Sinica, vol. 29, no. 8, pp. 2243-2247, 2009.

[19] G. P. Agrawal, Nonlinear Fiber Optics, Academic Press, 2007.

[20] C. Xu, Y. Dai, K. Xu, and X. Jia, "Spectral-filter-like effect of raman self frequency shift (RSFS) in all-normal-dispersion (ANDi) fiber laser," in Proceedings of the Asia Communications and Photonics Conference, ATh3A-83, Optical Society of America, Shanghai, China, November 2014.

[21] C. Xu, Y. Dai, and K. Xu, "Dissipative soliton (DS) pulse stability against ASE-noise in normal dispersion fiber laser based on a slow saturable absorber (SA)," in Proceedings of the Asia Communications and Photonics Conference (ACP '14), p. AF2C2, Optical Society of America, November 2014. 

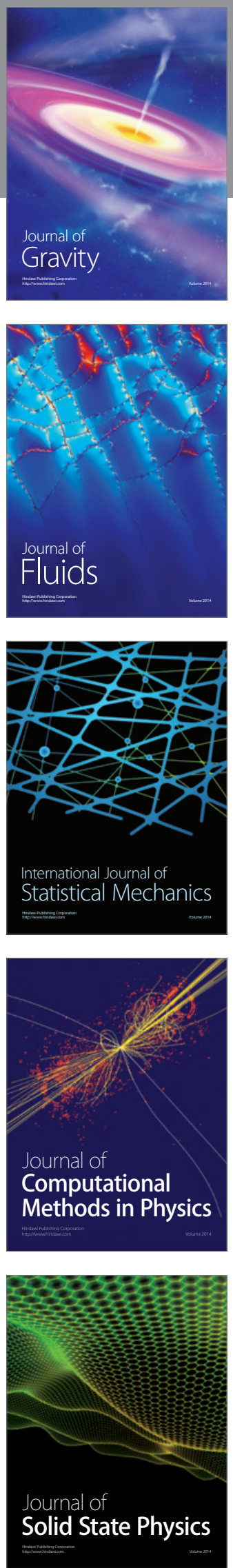

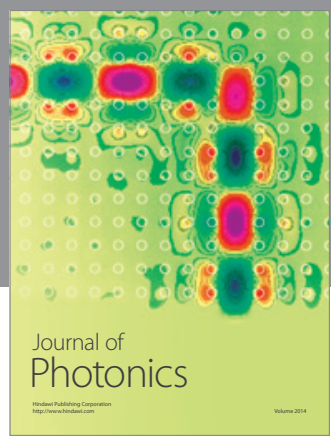

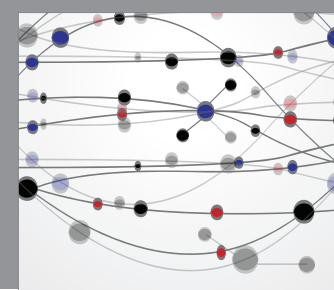

The Scientific World Journal

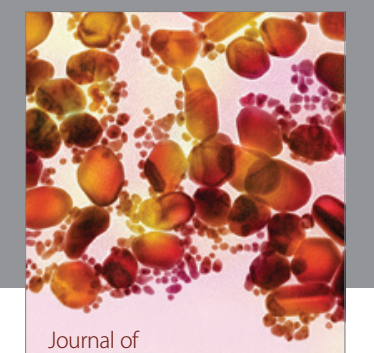

Soft Matter
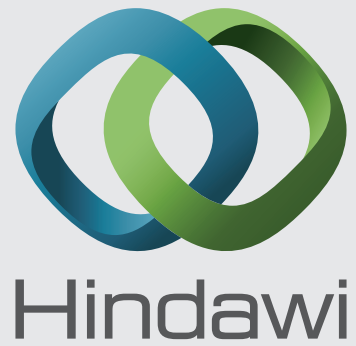

Submit your manuscripts at

http://www.hindawi.com
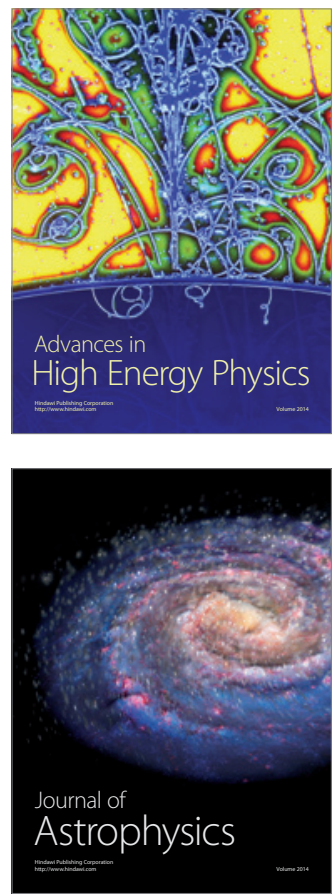
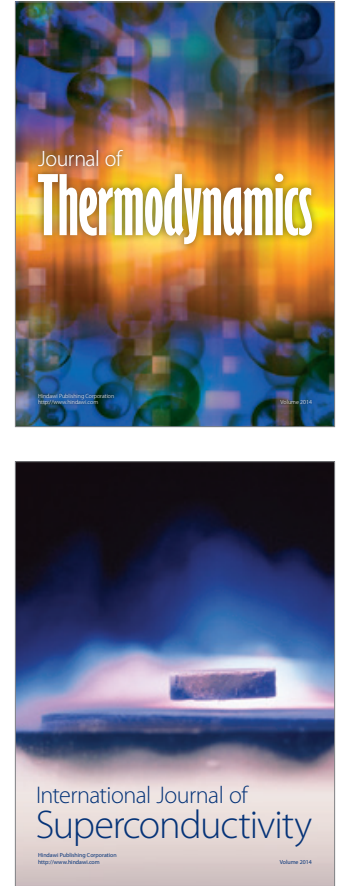
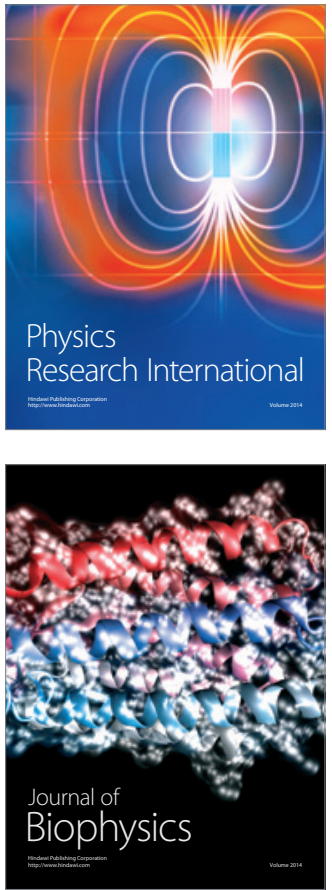
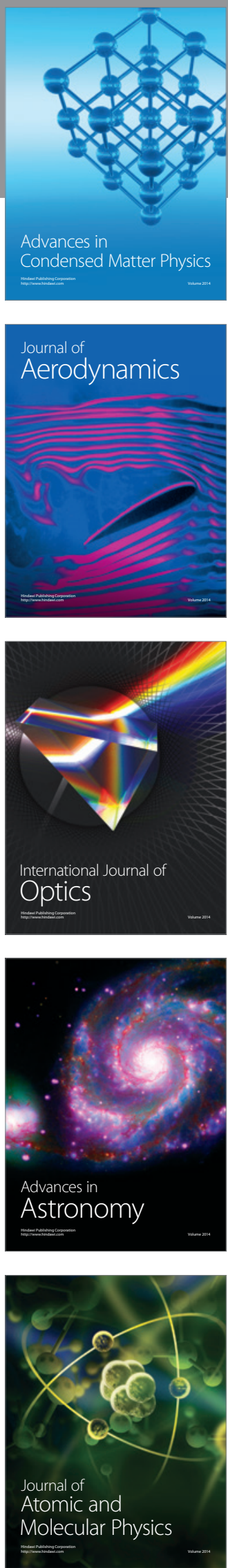